\title{
Analysis of the Prevalence of Headaches Subtypes in a Specialized Outpatient Clinic in a Medium-sized Municipality, Minas Gerais, Brazil
}

\author{
Estephanie Veronese Ribeiro ${ }^{(D)}$, Gustavo Alvarenga Rodrigues ${ }^{(D)}$, José Rafael Araújo e Costa (D), \\ Julia Rodrigues Pereira (D), Marcelo Rodrigues de Assis Júnior (iD, Mauro Eduardo Jurno
}

Faculdade de Medicina de Barbacena - FAME/FUNJOB

\section{$凶$}

Mauro Eduardo Jurno mejurno@gmail.com Praça Pres. Antônio Carlos, 8 . São Sebastiao, Barbacena - MG, 36202-336, mejurno@gmail. com, (032) $3331-7681$ (032) 99983-1266

Edited by

Marcelo Moraes Valença

Keywords:

Headache

Prevalence

Tertiary care center

Epidemiology

Migraine Disorders

Tension-Type Headache

\begin{abstract}
Objective

To evaluate the prevalence of different subtypes of headaches in a tertiary referral clinic for headache care in the city of Barbacena, Brazil.

Methods

A retrospective and descriptive cross-sectional study that evaluated the medical records of 130 patients previously seen by a neurologist in the headache clinic from January 2020 to March 2021.

Results

The mean age of patients was 42.5 years, most of them female (83\%). Primary headaches were the most common, with migraine found in $73 \%$ of cases and tension-type headache (TTH) in $17 \%$. Among secondary headaches, the most common subtype was headache attributed to a substance or its suppression (11\%), followed by cases such as headache due to cranial neuralgia and central causes of facial pain (3\%) and headache attributed to trauma or head and/or cervical injury (3\%). All cases in which there was an association of diagnoses of headache subtypes were found in patients with migraine (100\%).

Conclusion

This study defines the prevalence of headaches in a specialized outpatient clinic, confirming literature data that show migraine as the most common headache.
\end{abstract}




\section{Introduction}

- leadache is an important health problem in Brazil and worldwide because of its high incidence and prevalence, its socioeconomic impact, including its significant costs for medical services, its social and individual impact with possible reduction in quality of life and loss of productivity, and its high potential for chronification. ${ }^{1.5}$ It is estimated that $95 \%$ of men and $99 \%$ of women will have at least one episode of headache in their lifetime, of which approximately $40 \%$ will manifest with some regularity. ${ }^{6}$

The causes of headache are diverse, but the so-called primary headaches are more prevalent, which result from alterations in the central nervous system, such as tensiontype headache (TTH) and migraine. ${ }^{7}$ Headaches vary in frequency and intensity and can be as disabling as depression. 8,9

The high morbidity among sufferers of headaches such as migraine with or without aura, chronic migraine, and TTH, who seek routine and emergency medical care, shows the need for and the importance of epidemiological studies. According to Rouquayrol e Almeida Filho ${ }^{10}$ epidemiology plays an important role in improving the health of the population, since it provides the quantitative basis for the evaluation of prophylaxis measures and for the consistency of diagnostic hypothesis and the etiology of the disease. The International Association of Epidemiology establishes three main objectives for epidemiology: to describe the distribution and magnitude of health problems in human populations; to provide essential data for planning, implementing, and evaluating disease prevention, control, and treatment actions, as well as to establish priorities; and also, to identify etiological factors in the genesis of diseases. $^{11}$

According to a study that aimed to review previous literature on headache, the average prevalence of headache in Brazil is $70.6 \%$ (male $61.6 \%$ and female $77.8 \%$ ), of migraine $15.8 \%$ (male $9.0 \%$ and female $22.0 \%$ ), of $\mathrm{TTH}$ $29.5 \%$ (male $28.1 \%$ and female $30.3 \%$ ). ${ }^{12}$ Therefore, the incidence of the diagnostic types of headache in the present study is discordant compared to the frequency observed in the community, since the TTH characterizes the most prevalent headache in the general population, moreover, in large tertiary centers, a predominance of migraine is observed. ${ }^{9,13}$ This discrepancy is explained in part because TTH, although common, is less disabling, which decreases the demand for outpatient medical care, while in migraine, a type of headache that can be characterized by strong intensity and symptoms such as photo and phonophobia, scotomas, nausea and vomiting; greater demand for care is required. ${ }^{14}$

Thus, it is important to know the epidemiological profile of headaches seen in tertiary centers for a better diagnosis and for an effective treatment in primary and secondary care levels, aiming at health promotion and better management of pain. Therefore, this work is a descriptive study aimed at presenting the prevalence of the main headache disorders in a tertiary referral center for headache care in a mediumsized city in Minas Gerais (Barbacena), Brazil.

\section{Methods}

A cross-sectional, retrospective, and descriptive study was conducted to analyze the prevalence of the main headache disorders in a tertiary referral outpatient clinic for headache care in the city of Barbacena, a mediumsized city in the state of Minas Gerais- Brazil, by collecting data from patients' medical records. The study was carried out during the period from January 1, 2020, to February 28,2021 . Patients were previously evaluated by a neurologist and subsequently referred to the outpatient clinic. The patients' medical records were included in the study only after their first visit to the headache outpatient clinic.

For the classification of headaches, the criteria established by the 3rd Edition of the International Classification of Headache Disorders ${ }^{7}$ (ICHD-III) was used. The medical records contained the patients' anamnesis, collected by a specialist with experience in the treatment of headaches, ending with a diagnostic hypothesis, which was finally identified according to the International Headache Classification (ICHD-III). ${ }^{7}$

Parameters such as gender, age, adherence to treatment, imaging exams before or after the consultation, and use of abortive and/or prophylactic medication for the treatment of headaches were recorded.

\section{Analysis of the results}

A descriptive analysis of the variables used in the study was performed. The data collected through preestablished criteria for diagnosis of the various subtypes of headache with the neurologist's examination quantified the cases, and their data were transcribed to an electronic spreadsheet and processed in Excel software. For nominal or categorical variables, frequency distribution tables 
were made. For continuous variables, measures of central tendency and variability were used.

\section{Results}

Among the 130 records analyzed, it was observed a mean patient age of 42.55 years, with a predominance of females (83\%). Among these patients, the request for imaging exams was made to 29 patients $(22 \%)$, of which 20 had already presented previous exams, either requested in previous visits to the headache clinic or by other professionals.

Regarding the subtypes of headache, primary headaches were prevalent, with migraine without aura being the most common diagnosis (42\%), followed by TTH (17\%); migraine with aura (14\%); chronic migraine $(5 \%)$; cluster headache $(1 \%)$ and primary shooting headache (1\%). Regarding the other headache subtypes, which totaled $9 \%$ of the sample, the results were: $11 \%$ of the patients had the diagnosis of headache attributed to a substance or its suppression, $3 \%$ headache due to cranial neuralgias and central causes of facial pain, $3 \%$ headache attributed to trauma or head and/or neck injury, 1\% headache attributed to cranial and/or neck vascular disorder and $1 \%$ headache attributed to homeostasis disorder.

In addition, there was coexistence of headaches in the same patient, especially the association of primary headaches, mainly migraine, with headaches due to analgesic abuse, totaling $7.6 \%$ of patients. Table 1 illustrates the prevalence of headaches reported in this study. It can be observed that the prevalence of headaches exceeds the number of individuals studied, since a patient may present concomitantly more than one type of headache.

Table 1. Distribution of the number of headache cases, according to diagnosis $(n=130)$.

\begin{tabular}{|c|c|c|}
\hline Diagnósticos & $\mathrm{n}$ & $\%$ \\
\hline Migraine & 85 & 73 \\
\hline Migraine without aura & 49 & 42 \\
\hline Migraine with aura & 17 & 14 \\
\hline Chronic migraine & 6 & 5 \\
\hline Tension-type headache & 21 & 17 \\
\hline Salva headache & 1 & 1 \\
\hline Cranial neuralgias and central causes of facial pain & 4 & 3 \\
\hline Headache attributed to head and/or neck trauma or injury & 3 & 3 \\
\hline Headache attributed to cranial and/or cervical vascular disorder & 1 & 1 \\
\hline Headache attributed to homeostasis disorder & 1 & 1 \\
\hline Headache attributed to a substance or its suppression & 13 & 11 \\
\hline Primary cluster headache & 1 & 1 \\
\hline
\end{tabular}

It should be noted that adherence to treatment totaled 101 people $(86.3 \%)$ predominantly females $(84.2 \%)$. Of the total number of patients who adhered to the treatment, $75 \%$ had migraine headache, and $17 \%$ the $\mathrm{TTH}$ type.

All cases in which there was an association of headache subtype diagnoses in patients with migraine (100\%), most of them related to analgesic overuse headache $(71.4 \%)$ although it was also associated with: TTH (7.15\%), headache attributed to trauma (7.15\%), cluster headache (7.15\%) and primary shooting headache (7.15\%). The associations of these types of diagnoses with migraine can be seen in Table 2.

Table 2. Distribution of the number of cases with an association between migraine and other headache diagnoses $(n=14)$.

\begin{tabular}{|c|c|c|}
\hline \multicolumn{1}{|c|}{ Diagnoses } & $\mathrm{n}$ & $\%$ \\
\hline Headache attributed to a substance or its suppression & 10 & 71,4 \\
\hline Tension-type headache & 1 & 7,15 \\
\hline Salva headache & 1 & 7,15 \\
\hline Headache attributed to trauma or injury to the head and/or neck & 1 & 7,15 \\
\hline Primary shooting headache & 1 & 7,15 \\
\hline
\end{tabular}

\section{Discussion}

In 2016, TTH and migraine have reached third and sixth place, respectively, in the world's most prevalent diseases, affecting approximately 1.89 billion people and 1.04 billion people, according to the Global Burden of Disease Study $2016 .{ }^{15}$ In addition, the number of years lived with disability due to migraine reached second place, generating approximately 15 years of disability due to the disease. According to a study published in the Journal of Headache and Pain, migraine is the leading cause of disability under age $50 .{ }^{16}$

Epidemiological data highlight the relevance of headache as a symptom that directly impacts the quality of life of patients through physical suffering and emotional, economic, social, and labor losses. Notwithstanding, it is estimated that only $16 \%$ of patients with TTH and $56 \%$ of patients with migraine seek care from general practitioners, and among these, only $4 \%$ and $16 \%$, respectively, seek consultation with headache specialists. ${ }^{17}$

Estimating that only a small percentage of individuals with headache seek care from specialized doctors ${ }^{17}$, it is expected that the prevalence of headache in primary care and in the general population is divergent from the prevalence found in tertiary outpatient clinics. Although $\mathrm{CTT}$ is more prevalent in the general population, migraine 
is the most prevalent type of headache in tertiary outpatient clinics due to its characteristic of greater severity and greater impact on the quality of life of patients. ${ }^{6}$

According to a study in a tertiary outpatient clinic in Minas Gerais, primary headaches were the most prevalent, with migraine being found in $79.8 \%$ of cases and TTH in $20.4 \%$. Among the secondary ones, the most common type was headache due to excessive use of analgesics (16.6\%), followed by less common cases such as idiopathic intracranial hypertension. Chronic daily headache $(\mathrm{CDH})$ was present in $31.8 \%$ of the cases. ${ }^{18}$

Our study, conducted in a tertiary headache outpatient clinic in a medium-sized city in Minas Gerais with 130 patients (108 females and 22 males), showed that within the headache complaints (90\%; 117 individuals), 91\% (106 individuals) were diagnosed with primary headache. Among the primary headaches, the prevalence of migraine was $73 \%$ (85 individuals), and the prevalence of TTH among the primary headaches was 17\% (21 individuals). Our results show a very similar prevalence when compared to the one described in the literature in another tertiary outpatient clinic in Minas Gerais, with prevalence of migraine in $79.8 \%$ of cases and tension-type headache in $20.4 \% .^{18}$ In a Swiss study, migraine prevalence was $47.3 \%$, although $32.7 \%$ of patients had migraine in association with $\mathrm{TTH}^{19}$, situation found in only $1 \%$ (1 individual) of the patients in our study.

Tension-type headache was the second most frequent cause of headache in our study, with 17\% (21 individuals) of the primary headache cases in the tertiary outpatient clinic. A Chinese study showed a $23.7 \%$ prevalence of TTH in a tertiary clinic. ${ }^{20}$ A 2005 study conducted in Switzerland showed a $20 \%$ prevalence of TTH cases in a specialized headache clinic at a university hospital. ${ }^{19}$

There was coexistence of different headache disorders in the same patient, especially the association of primary headache disorders, mainly migraine, with analgesic abuse headache, totaling $7.6 \%$ of patients, a relatively lower rate when compared to another tertiary outpatient clinic of Minas Gerais. ${ }^{18}$ The prevalence of primary shooting headache was 1\% (1 patient), differing from the prevalence found in another tertiary center in Minas Gerais. ${ }^{18}$

The mean age of the patients in our study was 42.55 years; regarding sex, there was a predominance of females $(83.08 \%)$, in agreement with results found in another tertiary outpatient clinic in Minas Gerais. ${ }^{18}$ A study conducted in Switzerland showed a mean age of 38.5 years, with a predominance of females $(71,8 \%)^{19}$, while the Chinese study showed a mean age in a tertiary clinic of 42.8 years and a predominance of females $(66,9 \%) .{ }^{20}$

Our study had very similar results when evaluating the prevalence of migraine and TTH compared to another tertiary outpatient clinic in Brazil. Our study was also very similar to the studies conducted in tertiary outpatient clinics in Switzerland and China. Considering the impact of the disease on the lives of patients with headache, it is necessary that studies such as this one be conducted to highlight the relevant prevalence of headache subtypes, demonstrating the importance of diagnosis and the need for treatment to increase the quality of life and productivity of patients.

\section{Conclusion}

This study defines the prevalence of headaches in a specialized outpatient clinic, confirming literature data showing migraine as the most common headache subtype. Moreover, these data can help in the differential diagnosis of headache subtypes for the correct and early diagnosis and, consequently, ensure a good quality of life for those affected.

Conflict of interest statement: The authors declare that there are no conflicts of interest.

Funding: This work has no funding.

Estephanie Veronese Ribeiro

https://orcid.org/0000-0001-5432-9846

Gustavo Alvarenga Rodrigues

https://orcid.org/0000-0003-4700-2073

José Rafael Araújo e Costa

https://orcid.org/0000-0002-6167-7694

Julia Rodrigues Pereira

https://orcid.org/0000-0002-3863-5023

Marcelo Rodrigues de Assis Júnior

https://orcid.org/0000-0001-7105-0142

Mauro Eduardo Jurno

https://orcid.org/0000-0002-8743-9395

\section{References}

1. Rasmussen BK. Epidemiology and socio-economic impact of headache. Cephalalgia 1999;19 Suppl 25:20-23 Doi:10.1177/0333102499019s2505

2. Lipton RB and Bigal ME. Epidemiology of migraine in Latin America: an editorial. Headache 2005;45(2): 103- 
104 Doi:10.1111/j.1526-4610.2005.05023b.x

3. Schwartz BS, Stewart WF, Simon D and Lipton RB. Epidemiology of tension-type headache. Jama 1998;279(5):381-383 Doi:10.1001/jama.279.5.381

4. Rasmussen BK. Epidemiology of headache. Cephalalgia 1995;15(1):45-68 Doi:10.1046/j.14682982.1995.1501045.x

5. Berg J. Economic evidence in migraine and other headaches: a review. Eur J Health Econ 2004;5 Suppl 1:S43-54 Doi:10.1007/s10198-005-0288-z

6. Rasmussen BK, Jensen R, Schroll M and Olesen J. Epidemiology of headache in a general population--a prevalence study. J Clin Epidemiol 1991;44(11): 1147 1157 Doi:10.1016/0895-4356(91)90147-2

7. The International Classification of Headache Disorders, 3rd edition (beta version). Cephalalgia 2013; 33(9):629-808 Doi:10.1177/0333102413485658

8. Bigal ME, Fernandes LC, Moraes FA, Bordini CA and Speciali JG. Migraine prevalence and impact in employees of the clinical hospital of the medical school of Ribeirão Preto-USP. Arq Neuropsiquiatr 2000; 58 (2b): 431-436 Doi: 10.1590/s0004$282 \times 2000000300007$

9. Stovner L, Hagen K, Jensen R, Katsarava Z, Lipton R, Scher $A, \ldots$ Zwart JA. The global burden of headache: a documentation of headache prevalence and disability worldwide. Cephalalgia 2007;27(3):193210 Doi:10.1111/j.1468-2982.2007.01288.x

10. MZ R and FN A. Epidemiologia e saúde. 6 ed. Rio de Janeiro: Medsi; 2003.

11. JM L. A dictionary of epidemiology. 1 ed: Oxford university press Oxford, UK; 2001.

12. Bahra A, May A and Goadsby PJ. Cluster headache: a prospective clinical study with diagnostic implications. Neurology 2002;58(3):354-361 Doi:10.1212/ wnl.58.3.354

13. Silva-Júnior AA, Faleiros BE, Santos TM, Gómez RS and Teixeira AL. Relative frequency of headache types: a longitudinal study in the tertiary care. Ara Neuropsiquiatr 2010;68(6):878-881 Doi:10.1590/ s0004-282x2010000600009

14. Silva-Júnior AA, Moraes D, Rezende F, Pereira G, Morato $E$, Cunninghan $M$, . . . Pereira $S$. Freqüência dos tipos de cefaléias atendidos no pronto atendimento do Hospital das Clínicas da Universidade Federal de Minas Gerais. Migr Cefaleias 2008; 11 (2):67-72

15. Global, regional, and national incidence, prevalence, and years lived with disability for 328 diseases and injuries for 195 countries, 1990-2016: a systematic analysis for the Global Burden of Disease Study 2016. Lancet 2017;390(10100):1211-1259 Doi:10.1016/s0140-6736(17)32154-2

16. Steiner TJ, Stovner LJ, Vos T, Jensen R and Katsarava Z. Migraine is first cause of disability in under 50s: will health politicians now take notice? J Headache Pain 2018;19(1):17 Doi:10.1186/s10194-018-0846-2

17. Bigal ME, Bordini $C A$ and Speciali JG. Etiology and distribution of headaches in two Brazilian primary care units. Headache 2000;40(3):241-247 Doi: 10.1046/j. 1526-4610.2000.00035.x

18. Silva AA, Jr., Tavares RM, Lara RP, Faleiros BE, Gomez RS and Teixeira AL. Frequency of types of headache in the tertiary care center of the Hospital das Clínicas of the Universidade Federal de Minas Gerais, MG, Brazil. Rev Assoc Med Bras (1992) 2012;58(6):709713 Doi:10.1590/s0104-42302012000600017

19. Kozak S, Gantenbein AR, Isler H, Merikangas KR, Angst J, Gamma A and Agosti R. Nosology and treatment of primary headache in a Swiss headache clinic. J Headache Pain 2005;6(3):121-127 Doi:10.1007/s10194-005-0166-1

20. Li X, Zhou J, Tan G, Wang Y, Ran L and Chen L. Clinical characteristics of tension-type headache in the neurological clinic of a university hospital in China. Neurol Sci 2012;33(2):283-287 Doi:10.1007/ s10072-01 1-0675-4 